\title{
A Theoretical Investigation of Nuclear Reactions with Neutrons.
}

\author{
M. CrNi and S. Fubini \\ Istituto di Fisica dell'Università - Torino \\ Istituto Nazionale di Fisica Nucleare - Sezione di Trorino
}

\begin{abstract}
Gli Autori riferirono in questo "Seminario" i risultati di un loro lavoro originale, che era stato già presentato al Nuovo Oimento per la pubblicazione, e che comparve poi nel fascicolo di Luglio 1955 (pp. 75-89) dello stesso giornale; al quale quindi rimandiamo il lettore, mentre qui ci limitiamo a riportare il sommario. [N.d.R.]."
\end{abstract}

Summary. - A theory of the interaction of neutrons with complex nuolei is developed with the aim of obtaining a cross-section averaged over the resonances, to be compared with the results of the phenomenological model proposed by Festrach, PORTER and Weisstiopf (1). It is shown what kind of assumptions have to be introduced in order that the compound nucleus formation give rise to an absorption of the incident beam, irrespective of what happens after the compound nucleus decay. The problem is reduced to the determination of the complex index of refraetion of an indefinite nuelear matter, taking properly into account the effect of the Pauli principle. Subseruently this index of refraction has to be introduced into a one-body schrödinger equation with the correct boundary conditions at the nuclear wall. By assuming nuelear forces which fit the low energy two-body data, and an average binding energy of $8 \mathrm{MeV}$ per nucleon, an expression is derived for the absorption soefficient which is compared with the imaginary part of the FPW potential. At zero energy the absorption coefficient is just in the right range 0.03-0.05. For higher energies it becomes so large that already for 6.8 $\mathrm{MeV}$ the absorption is almost complete for medium sized nuclei. This agrees quite satisfactorily with experimental evidence.

(x) I. FKSHBACH, C. E. Porther and V. F. WersskopF: Phy. Rev., 96, 448 (1954) (to be denoted in the following as FPW). 